\title{
Antineoplastic activities of $\mathrm{Gd} @ \mathrm{C}_{82}(\mathrm{OH})_{22}$ nanoparticles: tumor microenvironment regulation
}

\author{
LI YiYe ${ }^{1}$, TIAN YanHuan ${ }^{1,2} \&$ NIE GuangJun ${ }^{1 *}$ \\ ${ }^{1}$ CAS Key Laboratory for Biomedical Effects of Nanomaterials \& Nanosafety, National Center for Nanoscience and Technology (NCNST), \\ Beijing 100190, China; \\ ${ }^{2}$ College of Pharmaceutical Science, Jilin University, Changchun 130021, China
}

Received September 4, 2012; accepted September 19, 2012

\begin{abstract}
Malignant tumors are complex organs consisting of tumor cells and their microenvironment. Increasing evidence has shown that the tumor microenvironment is critical to the initiation and progression of tumors. Rational design of tumor therapies via targeting the tumor microenvironment to inhibit tumor growth is thus becoming a consensus strategy. $\mathrm{Gd} @ \mathrm{C}_{82}(\mathrm{OH})_{22}$ nanoparticles, as novel endohedral hydroxylated metallofullerenes, have been demonstrated to be a potent antitumor nanomedicine via targeting multiple factors in the tumor microenvironment. $\mathrm{Gd} @ \mathrm{C}_{82}(\mathrm{OH})_{22}$ nanoparticles possess excellent biocompatibility and remarkable antineoplastic activity, as a result not of direct tumor cytotoxicity but of their diverse biological effects, including antioxidation, immune activation, angiogenesis inhibition, imprisoning cancer cells, and reversal of drug-resistance. In this article, we summarize the unique nanoscale physiochemical properties and the antineoplastic activities of $\mathrm{Gd}_{0} @ \mathrm{C}_{82}(\mathrm{OH})_{22}$ nanoparticles, and focus on the mechanisms underlying their regulation of the tumor microenvironment.
\end{abstract}

Gd@ $\mathrm{C}_{82}(\mathrm{OH})_{22}$, antineoplastic nanoparticles, tumor microenvironment

Citation: $\quad$ Li Y Y, Tian Y H, Nie G J. Antineoplastic activities of $\mathrm{Gd}_{\mathrm{C}} \mathrm{C}_{82}(\mathrm{OH})_{22}$ nanoparticles: tumor microenvironment regulation. Sci China Life Sci, 2012, 55: 884-890, doi: 10.1007/s11427-012-4387-7

Tumor therapy remains an important unresolved issue. The bottlenecks associated with traditional therapies have prompted the development of new treatments and a deeper understanding of the mechanisms of tumorigenesis and progression. Pathologically, cancer cells do not exist in isolation, but create a complex context with their microenvironment, which comprises proliferating tumor cells, tumor stroma, blood vessels, infiltrating inflammatory cells and a variety of associated stromal cells [1]. The tumor microenvironment operates complex mechanisms to maintain its dysregulated functions and to assist tumor cells to evade anticancer therapies. Ideally, to achieve maximal therapeutic efficacy, antitumor agents should not only inhibit and destroy the tumor cells, but also normalize the tumor mi-

*Corresponding author (email: niegj@ nanoctr.cn) croenvironment. Therapeutic agents that target the tumor microenvironment thus represent an important new direction for the development of novel and effective antitumor therapies. Some drugs targeting the tumor microenvironment, such as the angiogenesis inhibitor bevacizumab (Avastin), have already been approved by the US Food and Drug Administration for the treatment of several cancers $[2,3]$.

Engineered nanoparticles, including a variety of carbon nanomaterials, are emerging as robust platforms with immense potential for effective cancer therapy [4]. $\mathrm{Gd} @ \mathrm{C}_{82}(\mathrm{OH})_{22}$ is an endohedral metallofullerenol nanoparticle recently developed for biomedical applications, which has shown promising antineoplastic activity [5-9]. In contrast to other antitumor agents or nanomaterials, $\mathrm{Gd} @ \mathrm{C}_{82}(\mathrm{OH})_{22}$ nanoparticles do not kill tumor cells directly, but rather inhibit tumor growth 
and metastasis. Other studies have identified several underlying mechanisms closely related to various components of the tumor microenvironment [8-13]. The current review examines the unique antineoplastic activity of $\mathrm{Gd} @ \mathrm{C}_{82}(\mathrm{OH})_{22}$ nanoparticles via regulation of the tumor microenvironment, as a novel antitumor strategy associated with the physiochemical properties of the nanomaterial.

\section{Characterization}

$\mathrm{Gd} @ \mathrm{C}_{82}(\mathrm{OH})_{22}$ is an endohedral hydroxylated metallofullerene (metallofullerenol) with a unique structure and surface features. It possesses a virus-like nanostructure, consisting of a magnetic metal core of a gadolinium $(\mathrm{Gd})$ atom and a closed outer nanosheath of carbon atoms, which are further modified by about 22 outermost hydroxyl groups (Figure 1) [9]. Several studies have found that the composition and numbers of the outer groups are largely responsible for the bioactive properties of fullerenol molecules [5,7,14-18]. The $\mathrm{Gd} @ \mathrm{C}_{82}(\mathrm{OH})_{22}$ molecule is smaller than $2 \mathrm{~nm}$, but $\mathrm{Gd} @ \mathrm{C}_{82}(\mathrm{OH})_{22}$ tends to aggregate in aqueous solutions and forms dispersed $\mathrm{Gd} @ \mathrm{C}_{82}(\mathrm{OH})_{22}$ nanoparticles with an average diameter of about $100 \mathrm{~nm}$, which is a ubiquitous property identified in metallofullerenol studies [7]. $\mathrm{Gd} @ \mathrm{C}_{82}(\mathrm{OH})_{22}$ nanoparticles exhibit a high degree of stability and good dispersion in physiological solutions [11]. The surface functionalization of the metallofullerene overcomes the dissolvability limitation of conventional carbon nanomaterials, such as fullerenes and carbon tubes, and significantly broadens the potential biomedical applications of $\mathrm{Gd} @ \mathrm{C}_{82}(\mathrm{OH})_{22}$ nanoparticles. Further studies showed that $\mathrm{Gd} @ \mathrm{C}_{82}(\mathrm{OH})_{22}$ nanoparticles maintained their optical transparency and remained within a constant size range over a period of 1 month in physiological media, and no significant release of $\mathrm{Gd}^{3+}$ ions was detected by inductively coupled plasma-mass spectrometry within 6 days, indicating the intrinsic stability of $\mathrm{Gd} @ \mathrm{C}_{82}(\mathrm{OH})_{22}$ nanoparticles $[11,13]$. Some unique physicochemical properties of $\mathrm{Gd} @ \mathrm{C}_{82}(\mathrm{OH})_{22}$ nanoparticles have also been examined in subsequent studies [19-21].

\section{Biosafety}

Extensive studies have examined the biocompatibility and biosafety of $\mathrm{Gd} @ \mathrm{C}_{82}(\mathrm{OH})_{22}$ nanoparticles and have found no discernible toxic effects either in vitro or in vivo. These observations may be attributable to the water-soluble multi-hydroxyl groups on the surface. $\mathrm{Gd} @ \mathrm{C}_{82}(\mathrm{OH})_{22}$ nanoparticles do not affect either the viability or proliferation of human primary immune cells (B cells, T cells or macrophages) or tumor cells (hepatoma cells or lung carcinoma cells), nor induce their apoptosis [7,11]. The nanoparticles could be delivered to most tissues and organs in mice through the blood circulation, but barely penetrated the blood-brain barrier [10]. Standard histopathological examinations using hematoxylin-eosin (HE) staining revealed that $\mathrm{Gd} @ \mathrm{C}_{82}(\mathrm{OH})_{22}$ nanoparticles induced no abnormal pathological changes in the liver, spleen, kidney, heart, brain, or lung of treated mice, compared with untreated controls [7]. Comparison of the biodistributions of $\mathrm{Gd} @ \mathrm{C}_{82}(\mathrm{OH})_{22}$ nanoparticles and metallic $\mathrm{Gd}^{3+}$ suggest that the fullerene cage is not destroyed during metabolism in mice, and the internal $\mathrm{Gd}^{3+}$ is thus not liberated into the tissues [10]. In addition, $\mathrm{Gd} @ \mathrm{C}_{82}(\mathrm{OH})_{22}$ nanoparticles caused no abnormal behavior, deaths or other systemic toxicities in tumor-bearing mice $[10,11]$.

In vivo toxicological studies are critical for the clinical development of all investigational nanomaterials. In addition to the traditional rodent animal models used in toxicity studies, the nematode Caenorhabditis elegans (C. elegans) has also been used to further evaluate the potential adverse in vivo effects of $\mathrm{Gd} @ \mathrm{C}_{82}(\mathrm{OH})_{22}$ nanoparticles on organisms under normal and stress conditions [22]. Treatment with $\mathrm{Gd} @ \mathrm{C}_{82}(\mathrm{OH})_{22}$ nanoparticles up to $10 \mathrm{mg} \mathrm{mL}^{-1}$ within one generation (short-term) and six generations (long-term) resulted in no significant decreases in longevity or thermotolerance in C. elegans. In addition, individual growth and development, feeding behavior and reproductive capability also remained relatively unchanged after exposure to the nanoparticles. Overall, these data demonstrate that $\mathrm{Gd} @ \mathrm{C}_{82}(\mathrm{OH})_{22}$ nanoparticles are well tolerated by $C$. elegans with no apparent toxic effects in either adult or young worms [22].

All biosafety studies conducted so far have demonstrated that $\mathrm{Gd} @ \mathrm{C}_{82}(\mathrm{OH})_{22}$ nanoparticles have a high safety profile, thus laying the foundations for the further development of biomedical applications. Furthermore, $\mathrm{Gd} @ \mathrm{C}_{82}(\mathrm{OH})_{22}$ nanoparticles have also recently been developed as a superior contrast agent for magnetic resonance imaging [5,6], accompanying their high efficiency for antitumor growth.

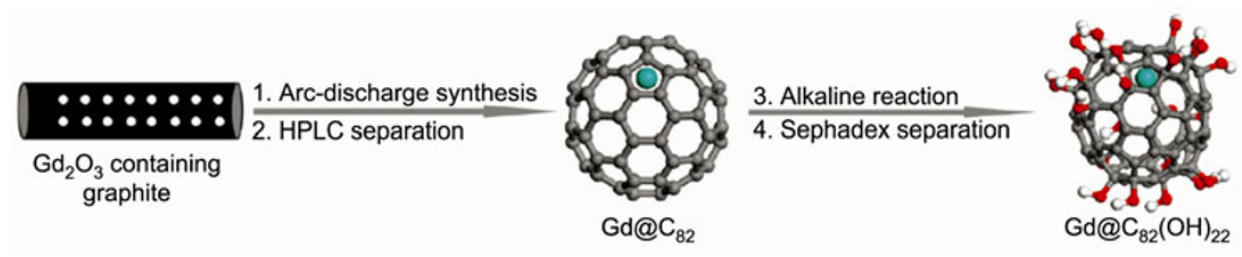

Figure 1 Synthesis and structure of $\mathrm{Gd} @ \mathrm{C}_{82}(\mathrm{OH})_{22}$. Reproduced with permission [9]. 


\section{Tumor suppression}

\subsection{Antineoplastic activity of $\mathbf{G d} @ \mathrm{C}_{82}(\mathrm{OH})_{22}$}

Various lines of evidence have confirmed antineoplastic tumor-suppressing activity of $\mathrm{Gd} @ \mathrm{C}_{82}(\mathrm{OH})_{22}$ nanoparticles and identified their molecular mechanisms since the nanoparticles were reported to prevent tumor proliferation in a rodent hepatocellular carcinoma model in 2005 [7]. The nanoparticles, at a dose as low as $10^{-7} \mathrm{~mol} \mathrm{~kg}{ }^{-1}$, inhibit hepatocellular damage in association with about $60 \%$ inhibition of tumor growth [7]. Although the molar dosage of $\mathrm{Gd} @ \mathrm{C}_{82}(\mathrm{OH})_{22}$ nanoparticles was approximately $1 / 500$ that of the antineoplastic agent cyclophosphamide, their efficiency was much higher under the same experimental conditions, and no observable damage to any important organs was reported [7]. Furthermore, $\mathrm{Gd} @ \mathrm{C}_{82}(\mathrm{OH})_{22}$ nanoparticles significantly inhibited lung tumor growth, with average inhibition rates for nanoparticles administered at $10^{-7} \mathrm{~mol}$ $\mathrm{kg}^{-1}$ and $5 \times 10^{-7} \mathrm{~mol} \mathrm{~kg}{ }^{-1} 40.2 \%$ and $45.3 \%$, respectively [11]. $\mathrm{Gd} @ \mathrm{C}_{82}(\mathrm{OH})_{22}$ nanoparticles could also efficiently prohibit liver, kidney and lung damage in tumor-bearing mice, and the organ functions were significantly improved $[7,10,23]$. The efficacy of treatment with $\mathrm{Gd} @ \mathrm{C}_{82}(\mathrm{OH})_{22}$ nanoparticles in breast-tumor-bearing nude mice was comparable to the commonly used clinical anticancer drug paclitaxel, with no detectable side effects [9]. Moreover, $\mathrm{Gd} @ \mathrm{C}_{82}(\mathrm{OH})_{22}$ nanoparticles could reactivate defective endocytosis of cisplatin in cisplatin-resistant (CP-r) cells, and tumor resistance to cisplatin was overcome by treatment with a combination of $\mathrm{Gd} @ \mathrm{C}_{82}(\mathrm{OH})_{22}$ nanoparticles and cisplatin, both in vitro and in vivo [24].

\subsection{Antineoplastic mechanisms of $\mathrm{Gd} @ \mathrm{C}_{82}(\mathrm{OH})_{22}$}

The high antitumor efficiency of $\mathrm{Gd} @ \mathrm{C}_{82}(\mathrm{OH})_{22}$ nanoparticles is not the result of traditional cytotoxic effects, but rather their direct regulation of the tumor microenvironment. The nanoparticles do not kill tumor cells directly, and less than $0.05 \%$ of the exposed dose of $\mathrm{Gd} @ \mathrm{C}_{82}(\mathrm{OH})_{22}$ nanoparticles was distributed in the tumor tissues in tumor-bearing mice $[7,11]$.

\subsubsection{Reactive oxygen species-scavenging/antioxidative function}

Oxidative stress is a response to the abnormal metabolism of reactive oxygen species (ROS) in aerobic organisms, and is considered an important mechanism in carcinogenesis. ROS can react with most biological macromolecules to cause oxidative damage and participate in building the intricate tumor microenvironment. Regulating oxidative stress is thus an important part of regulating the tumor microenvironment, and $\mathrm{Gd} @ \mathrm{C}_{82}(\mathrm{OH})_{22}$ nanoparticles can play an important role in regulating the oxidative defense system in vivo. These nanoparticles have been shown to inhibit the proliferation of solid tumors and decrease the activities of hepatic superoxide dismutase, glutathione peroxidase, glutathione S-transferase and catalase, as well as the levels of reduced glutathione, protein-bound thiols and malondialdehyde, which are responsible for catalyzing the production of ROS in vivo [10]. All major physiologically relevant ROS, including superoxide radical anion, hydroxyl radical and singlet oxygen, can also be scavenged directly by $\mathrm{Gd} @ \mathrm{C}_{82}(\mathrm{OH})_{22}$ nanoparticles [8,23]. Inhibition of lipid peroxidation and mitochondrial damage are also observed after treatment with $\mathrm{Gd} @ \mathrm{C}_{82}(\mathrm{OH})_{22}$ nanoparticles both in vitro and in vivo [23]. These results indicate that $\mathrm{Gd} @ \mathrm{C}_{82}(\mathrm{OH})_{22}$ nanoparticles can exert antitumor activity via regulating oxidative stress in the tumor microenvironment.

\subsubsection{Cellular immunity activation}

Upregulation of the inflammatory immune responses is a classical strategy for rapid tumor proliferation and metastasis. Gd@ $\mathrm{C}_{82}(\mathrm{OH})_{22}$ nanoparticles could trigger anti-inflammatory immune responses by activating lymphocytes and macrophages both in vitro and in vivo. $\mathrm{Gd} @ \mathrm{C}_{82}(\mathrm{OH})_{22}$ nanoparticles showed a strong capacity for improving immunity and interfering with hepatocellular carcinoma invasion in normal muscle cells and tumor-bearing mice [7]. Enhanced immunity can induce various effects such as lymphocyte proliferation and neutrophil migration into tumor tissues, and lymphocyte hyperplasia (lymphopoiesis), and aggregated follicles clearly appeared around the transplanted tumor tissues in $\mathrm{Gd} @ \mathrm{C}_{82}(\mathrm{OH})_{22}$ nanoparticle-treated animals. $\mathrm{Gd} @ \mathrm{C}_{82}(\mathrm{OH})_{22}$ nanoparticles can also inhibit the growth of lung carcinomas through specific immunomodulatory effects on T cells and macrophages, including polarizing the cytokine balance towards Th1 cytokines, decreasing the production of Th2 cytokines, and increasing the production of Th1 cytokines [11]. The nanoparticles stimulated immune cells to release tumor necrosis factor- $\alpha$, which plays an important role in cellular immune processes. Dendritic cells (DCs) also play a pivotal role in host immune defense, including inhibiting tumorigenesis. $\mathrm{Gd} @ \mathrm{C}_{82}(\mathrm{OH})_{22}$ nanoparticles are a potent activator of DCs towards the Th1 immune response. They could induce the phenotypic maturation of DCs by stimulating their production of cytokines, including interleukin-12p70, upregulating DC co-stimulatory and MHC molecules, and switching DCs from a CCL5responsive to a CCL19-responsive phenotype [12]. Overall, the results of these experiments suggest that $\mathrm{Gd} @ \mathrm{C}_{82}(\mathrm{OH})_{22}$ nanoparticles can effectively regulate the tumor microenvironment by regulating the host immune system to scavenge tumor cells (Figure 2) [11].

\subsubsection{Angiogenesis inhibition}

Tumor blood vessels are essential for the growth and survival of solid tumors. They also support the metastasis of tumor cells in the tumor microenvironment. Angiogenesis 


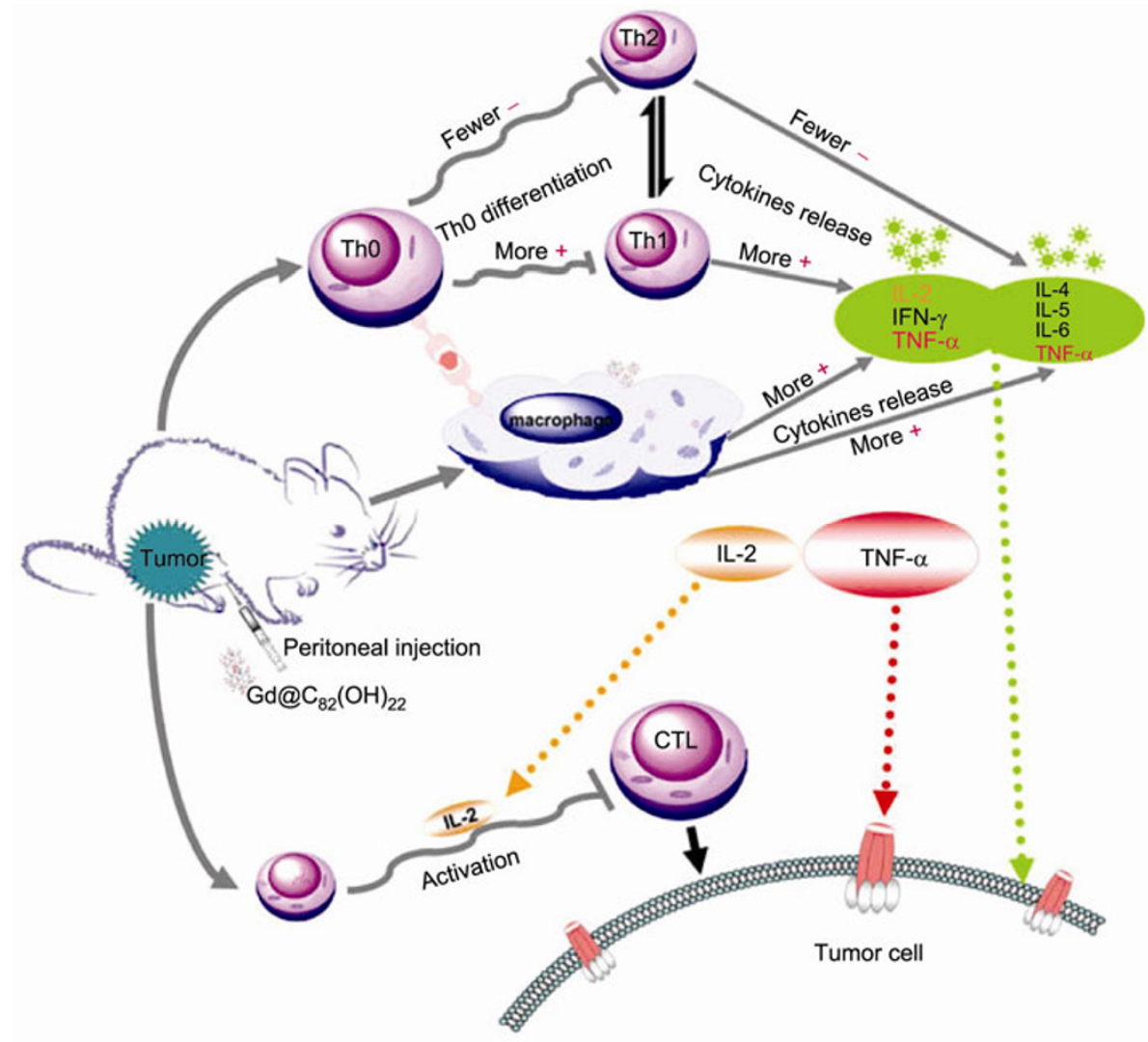

Figure 2 Possible immune-related mechanisms of $\mathrm{Gd} @ \mathrm{C}_{82}(\mathrm{OH})_{22}$ nanoparticles in inhibiting tumor growth. Reproduced with permission [11].

inhibition is emerging as an important strategy in the development of antitumor therapies, in addition to therapies aimed at directly killing or removing tumor cells. A variety of angiogenic inhibitors have been approved or are currently under investigation in clinical studies. However, the efficiencies of most small molecule and peptide inhibitors and antibody-based regulators are reduced in clinical studies, because their narrow inhibition spectra increase the risk of drug-resistance and side effects associated with a lack of specificity.

$\mathrm{Gd} @ \mathrm{C}_{82}(\mathrm{OH})_{22}$ nanoparticles represent a new 'particulate form' of angiogenesis inhibitor, with more favorable features than the traditional 'molecular form' inhibitors. $\mathrm{Gd} @ \mathrm{C}_{82}(\mathrm{OH})_{22}$ nanoparticles have a wide inhibition spectrum of more than 10 angiogenic factors [9]. At the mRNA level, the group of genes encoding fibroblast growth factor (FGF)1, FGF6, FGF receptor 3; chemokine (CXC motif) ligand (CXCL) 1, CXCL2, CXCL5, matrix metalloproteinase (MMP) 19, MMP2, MMP9, laminin subunit $\alpha-5$, and transforming growth factor (TGF) b1 and TGFb2 were significantly and simultaneously downregulated by treatment with $\mathrm{Gd} @ \mathrm{C}_{82}(\mathrm{OH})_{22}$ nanoparticles [9]. These promising observations have been further confirmed at the protein level [9]. The migration of human microvascular endothelial cells was suppressed by more than $80 \%$ in vitro, and the microvessel density in tumors was decreased by more than $40 \%$ after nanoparticle treatment [9]. Furthermore, $\mathrm{Gd} @ \mathrm{C}_{82}(\mathrm{OH})_{22}$ nanoparticles largely reduced tumor blood perfusion and efficiently lowered the speed of blood flow to tumor tissues by about $40 \%$ [9]. The results of in vivo experiments showed that $\mathrm{Gd} @ \mathrm{C}_{82}(\mathrm{OH})_{22}$ nanoparticles potently inhibited tumor growth, with a tumor-inhibition rate higher than the clinical breast-cancer-specific chemotherapeutic agent paclitaxel, at a dosage of $\mathrm{Gd} @ \mathrm{C}_{82}(\mathrm{OH})_{22}$ nanoparticles one-third the dose of paclitaxel [9]. At the same time, $\mathrm{Gd} @ \mathrm{C}_{82}(\mathrm{OH})_{22}$ nanoparticles did not interfere with normal blood vessels, and no changes in the morphology of capillary vessels in normal tissue were seen, including no pronounced abnormalities such as gaps, disfigurements or breaches in the cell layer in blood vessels. These findings confirm the angiogenesis-inhibitory activity of $\mathrm{Gd} @ \mathrm{C}_{82}(\mathrm{OH})_{22}$ nanoparticles in the tumor microenvironment (Figure 3) [9].

\subsubsection{Imprisoning rather than poisoning of cancer cells}

Tumor metastasis is a major contributor to over $90 \%$ of cancer-associated deaths. MMPs are critical regulators secreted mainly by tumor-associated macrophages in the tumor microenvironment. MMPs degrade extracellular matrix proteins and ease tumor invasiveness at the initial stage of tumor metastasis. Downregulation of MMP expression in the tumor microenvironment is known to be a major contributory factor in the decreased metastatic potential of tu- 


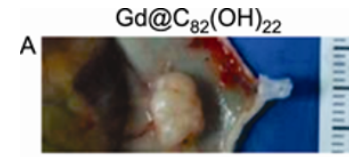

$\mathrm{Gd} @ \mathrm{C}_{82}(\mathrm{OH})_{22}$
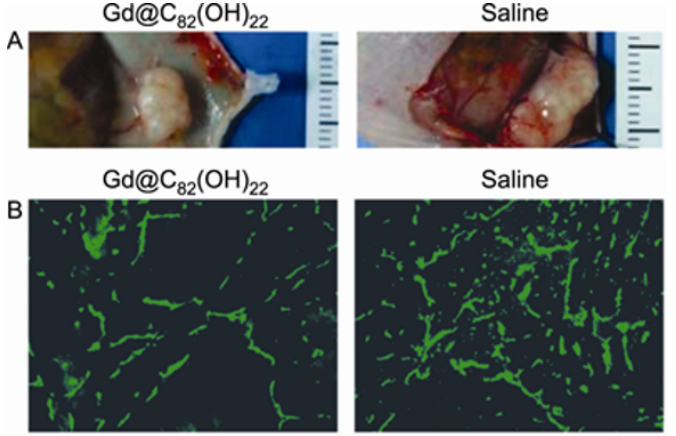

Saline

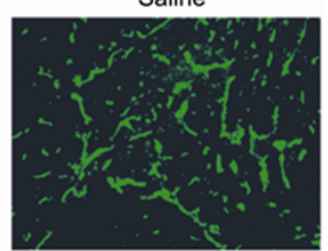

Figure 3 In vivo anti-angiogenic effects of $\mathrm{Gd} @ \mathrm{C}_{82}(\mathrm{OH})_{22}$ nanoparticles in mice. A, Morphology of tumor tissue treated with $\mathrm{Gd} @ \mathrm{C}_{82}(\mathrm{OH})_{22}$ nanoparticles. B, Tumor tissues stained for CD31 immunohistochemistry showing reduced blood vessel density. Reproduced with permission [9].

mors.

High-throughput gene-array analysis studies have revealed that members of the MMP family are strongly inhibited in response to $\mathrm{Gd} @ \mathrm{C}_{82}(\mathrm{OH})_{22}$ nanoparticle treatment [9]. Further studies revealed that $\mathrm{Gd} @ \mathrm{C}_{82}(\mathrm{OH})_{22}$ nanoparticles potently inhibited the production of MMPs at both the mRNA and protein levels in an MDA-MB-231 cancer cell-macrophage co-culture system, and cancer cell invasion was accordingly restrained in vitro [13]. In a tissue-invasion model, Gd@ $\mathrm{C}_{82}(\mathrm{OH})_{22}$ nanoparticles significantly suppressed tumor metastasis at the ectopic site, along with decreases in MMP-9 and MMP-2 expression of more than $90 \%$ and $60 \%$, respectively. Interestingly, histological examination by Van Gieson staining of primary subcutaneous tumors derived from MDA-MB-231 cells demonstrated a thick fibrous layer on the tumor surface in $\mathrm{Gd} @ \mathrm{C}_{82}(\mathrm{OH})_{22}$ nanoparticle-treated mice, but not in saline-treated mice [13]. In an- other breast-cancer model derived from MCF-7 cells, the fibrous layer surrounding the surface of the tumor was about $578 \mu \mathrm{m}$ thick in the nanoparticle-groups, compared with about $32 \mu \mathrm{m}$ in the saline-treated group. At the same time, no remarkable deposition of fibrous tissue was observed in any major organs other than the tumors [13]. In a bloodstream-transfer model in which MDA-MB-231 cancer cells were injected directly into the tail vein of nude mice, intraperitoneal Gd@ $\mathrm{C}_{82}(\mathrm{OH})_{22}$ nanoparticles injected daily for 6 weeks significantly inhibited the establishment of tumor foci in the lung [13]. Tumors were detected in the lungs at about 21 days after cancer cell injection in the saline group, but no cancers were detected in mice treated with $\mathrm{Gd} @ \mathrm{C}_{82}(\mathrm{OH})_{22}$ nanoparticles until day 42. Histological examination revealed the presence of numerous tumor foci in saline-treated controls, and minimal signs of tumors in the nanoparticle-treated group, highlighting the efficacy of $\mathrm{Gd} @ \mathrm{C}_{82}(\mathrm{OH})_{22}$ nanoparticles. These findings suggest that $\mathrm{Gd} @ \mathrm{C}_{82}(\mathrm{OH})_{22}$ nanoparticles operate via a new antitumor mechanism. The formation of a thick fibrous cage may act as a 'prison', confining invasive tumor cells to their primary site and thus effectively inhibiting the tumor via imprisoning, rather than poisoning the tumor cells (Figure 4) [13].

\subsubsection{Restoring defective endocytosis}

Chemotherapy remains a mainstream treatment in clinical tumor therapy, but its high toxicity and the easy development of drug-resistance greatly impede its applications. The loss of sensitivity of cells to chemotherapeutic drugs is a result of interactions between tumor cells and their microenvironment. Cisplatin is a commonly used chemotherapeutic agent for a number of epithelial malignancies; however,
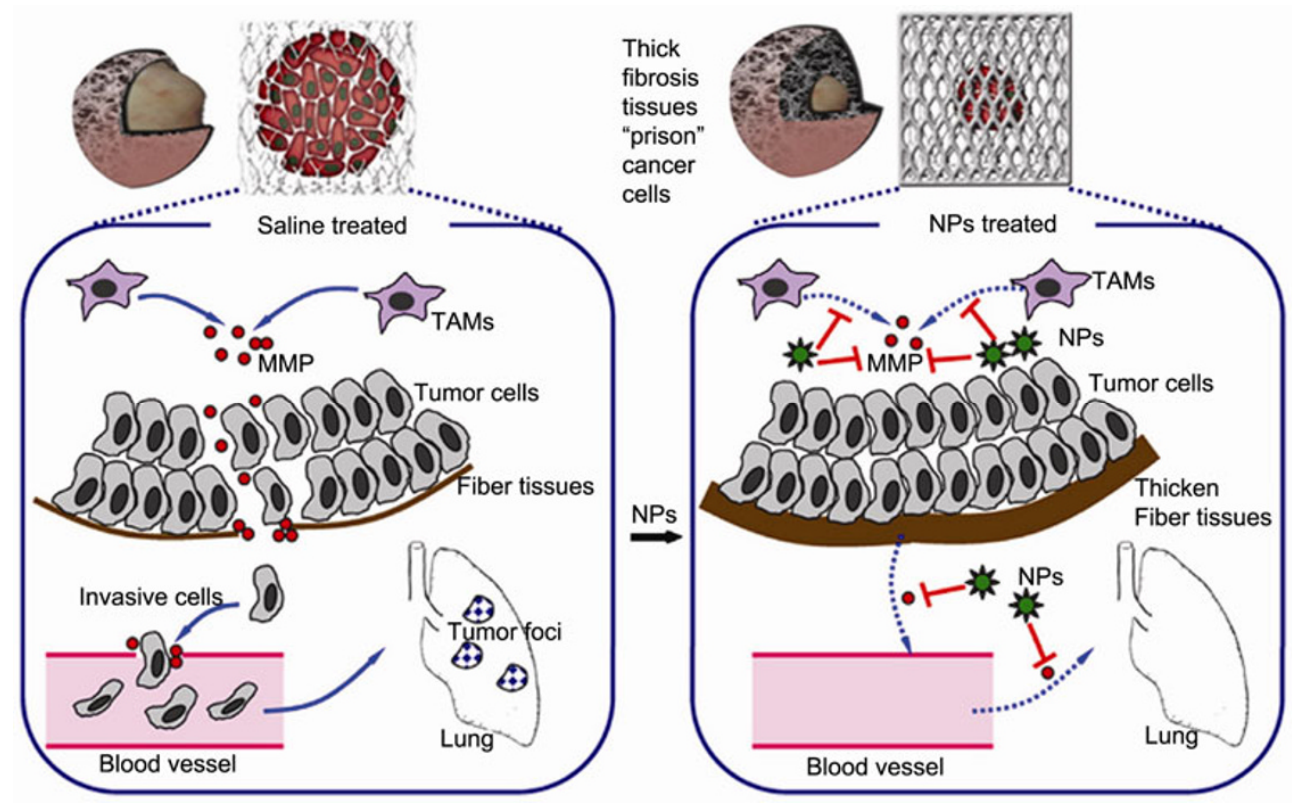

Figure 4 Schematic representation of possible anti-metastatic mechanism of $\mathrm{Gd} @ \mathrm{C}_{82}(\mathrm{OH})_{22}$ nanoparticles (NPs) mainly via a MMP-inhibition process. The thick fibrous cage may act as a 'prison' to confine invasive cancer cells within the primary site. Reproduced with permission [13]. 
the appearance of CP-r human prostate cancer cells seriously reduces its clinical efficacy. CP-r cells show defective endocytosis, which may lead to diminished accumulation of cisplatin and thus confer resistance. $\mathrm{Gd} @ \mathrm{C}_{82}(\mathrm{OH})_{22}$ nanoparticles could restore transferrin-mediated endocytosis, thus circumventing the acquired resistance of CP-r cells by enhancing uptake of cisplatin (Figure 5) [24]. Exposure of CP-r cells to cisplatin in the presence of $\mathrm{Gd} @ \mathrm{C}_{82}(\mathrm{OH})_{22}$ nanoparticles not only decreased the numbers of surviving CP-r cells, but also inhibited growth of CP-r tumors in athymic nude mice [24]. Measurement of cellular viability and cytotoxicity indicated that the nanoparticles were nontoxic to cancer cells in vitro, yet could successfully inhibit tumor growth in conjunction with cisplatin in CP-r tumors in vivo (Figure 5) [24].

\section{Prospects}

Recent advances in cancer nanotechnology along with its wide application in biomedicine offer new potential cancer

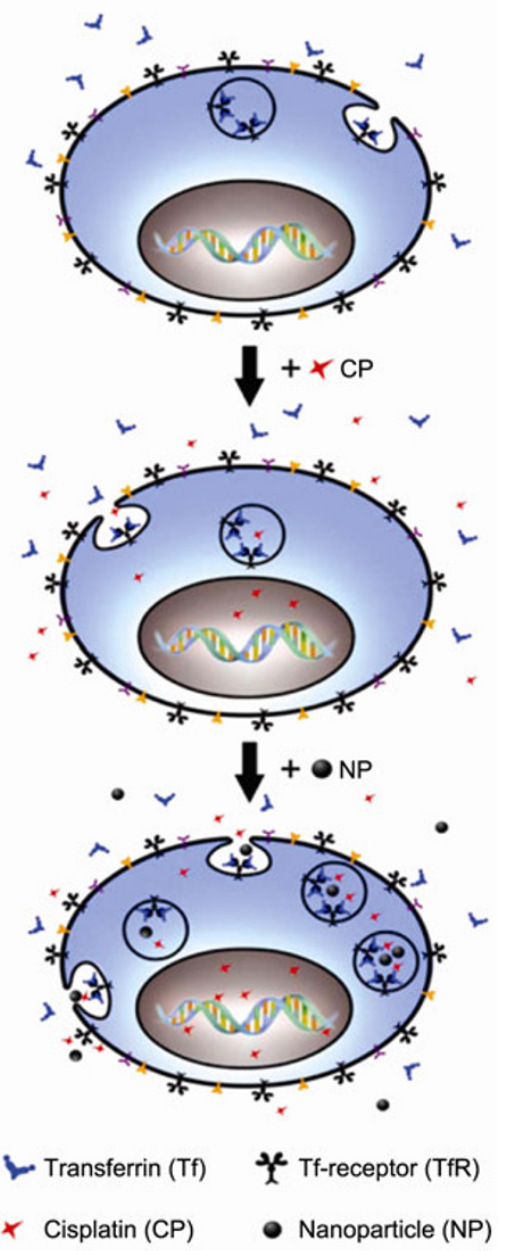

Figure 5 Schematic illustration of mechanism whereby $\mathrm{Gd} @ \mathrm{C}_{82}(\mathrm{OH})_{22}$ nanoparticles could increase CP-r cell sensitivity to cisplatin. Reproduced with permission [24]. therapeutic strategies. $\mathrm{Gd} @ \mathrm{C}_{82}(\mathrm{OH})_{22}$ nanoparticles, among the many biomedical nanomaterials with distinct antitumor properties, hold great promise associated with their novel antitumor activities via regulation of the tumor microenvironment. Growing awareness of the importance and complexity of the tumor microenvironment means that rational drug combinations targeting the whole 'tumor organ', namely both tumor cells and their microenvironment, are emerging as the mainstream of cancer therapy. $\mathrm{Gd} @ \mathrm{C}_{82}(\mathrm{OH})_{22}$ nanoparticles, as an excellent example of 'particulate medicine', represent a promising new opportunity for relieving the suffering of cancer patients in the near future.

1 Bissell M J, Radisky D. Putting tumours in context. Nat Rev Cancer, 2001, 1: 46

2 Bissell M J, Hines W C. Why don't we get more cancer? A proposed role of the microenvironment in restraining cancer progression. Nat Med, 2011, 17: 320-329

3 Avastin (bevacizumab) Information. U.S. Food and Drug Administration. http://www.fda.gov/Drugs/DrugSafety/Postmarket-DrugSafetyInformationforPatientsandProviders/ucm193900.htm

4 Yan L, Zhao F, Li S F, et al. Low-toxic and safe nanomaterials by surface-chemical design, carbon nanotubes, fullerenes, metallofullerenes, and graphenes. Nanoscale, 2011, 3: 362-382

5 Qu L, Cao W B, Xing G M, et al. Study of rare earth encapsulated carbon nanomolecules for biomedical uses. J Alloy Compd, 2006, 408/412: 400-404

6 Xing $\mathrm{G} \mathrm{M}$, Yuan $\mathrm{H}$, He R, et al. The strong MRI relaxivity of paramagnetic nanoparticles. J Phys Chem B, 2008, 112: 6288-6291

7 Chen C Y, Fang X H. Multi hydroxylated $\left[\mathrm{Gd} @ \mathrm{C}_{82}(\mathrm{OH})_{22}\right]_{\mathrm{n}}$ nanoparticles: antineoplastic activity of high efficiency and low toxicity. Nano Lett, 2005, 5: 2050-2057

8 Yin J J, Lao F, Fu P P, et al. The scavenging of reactive oxygen species and the potential for cell protection by functionalized fullerene materials. Biomaterials, 2009, 30: 611-621

9 Meng H, Xing G M, Sun B Y, et al. Potent angiogenesis inhibition by the particulate form of fullerene derivatives. ACS Nano, 2010, 4: 2773-2783

10 Wang $\mathrm{J} \mathrm{X}$, Chen $\mathrm{C}, \mathrm{Li} \mathrm{B}$, et al. Antioxidative function and biodistribution of $\left[\mathrm{Gd} @ \mathrm{C}_{82}(\mathrm{OH})_{22}\right]_{\mathrm{n}}$ nanoparticles in tumor-bearing mice. Biochem Pharmacol, 2006, 71: 872-881

11 Liu Y, Jiao F, Qiu Y, et al. The effect of $\mathrm{Gd} @ \mathrm{C}_{82}(\mathrm{OH})_{22}$ nanoparticles on the release of Th1/Th2 cytokines and induction of TNF-alpha mediated cellular immunity. Biomaterials, 2009, 30: 3934-3945

12 Yang D, Zhao Y L, Guo H, et al. $\left[\mathrm{Gd} @ \mathrm{C}_{82}(\mathrm{OH})_{22}\right]_{\mathrm{n}}$ nanoparticles induce dendritic cell maturation and activate Th1 immune responses. ACS Nano, 2010, 4: 1178-1186

13 Meng H, Xing G, Blanco E, et al. Gadolinium metallofullerenol nanoparticles inhibit cancer metastasis through matrix metalloproteinase inhibition: imprisoning instead of poisoning cancer cells. Nanomedicine: NBM, 2012, 8: 136-146

14 Tang J, Xing G, Yuan $\mathrm{H}$, et al. Tuning electronic properties of metallic atom in bondage to a nanospace. J Phys Chem B, 2005, 109: 8779-8785

15 Tang J, Xing G, Zhao Y, et al. Periodical variation of electronic properties in polyhydroxylated metallofullerene materials. Adv Mater, 2006, 18: 1458-1462

16 Cheng Y, Liu K M, Xing G, et al. Study of multihydroxylated processes of Gd@ $\mathrm{C}_{82}$ by ICP-MASS. J Radioanal Nucl Chem, 2007, 272: 537-540

17 Tang J, Xing G M, Zhao F, et al. Modulation of structural and electronic properties of fullerene and metallofullerenes by surface chemical modifications. J Nanosci Nanotechnol, 2007, 7: 1085-1101 
18 Yue D M, Bai X J, Zhao S X, et al. First endohedral metallofullerene-containing polymer: preparation and characterization of Gd@ $\mathrm{C}_{82}$-Polystyrene. J Phys Chem C, 2010, 114: 7631-7636

19 Tang J, Xing G, Yuan H, et al. 5p Electronic properties of Gd in $\mathrm{Gd} @ \mathrm{C}_{82}(\mathrm{OH})_{\mathrm{x}}$ studied by synchrotron radiation XPS. J Radioanal Nucl Chem, 2007, 272: 307-310

20 Tang J, Xing G, Zhao Y, et al. Switchable semiconductive property of the polyhydroxylated metallofullerene. J Phys Chem B, 2007, 111: 11929-11934

21 Zhao S X, Zhang J, Dong J Q, et al. Scanning tunneling microscopy investigation of substrate-dependent adsorption and assembly of metallofullerene Gd@ $\mathrm{C}_{82}$ on $\mathrm{Cu}(111)$ and $\mathrm{Cu}(100)$. J Phys Chem C, 2011, 115: 6265-6268

22 Zhang W D, Sun B, Zhang L, et al. Biosafety assessment of $\mathrm{Gd} @ \mathrm{C}_{82}(\mathrm{OH})_{22}$ nanoparticles on Caenorhabditis elegans. Nanoscale, 2011, 3: 2636-2641

23 Yin $\mathrm{J}$ J, Lao $\mathrm{F}$, Meng $\mathrm{J}$, et al. Inhibition of tumor growth by endohedral metallofullerenol nanoparticles optimized as reactive oxygen species scavenger. Mol Pharmacol, 2008, 74: 1132-1140

24 Liang X J, Meng H, Wang Y, et al. Metallofullerene nanoparticles circumvent tumor resistance to cisplatin by reactivating endocytosis. Proc Natl Acad Sci USA, 2010, 107: 7449-7454

Open Access This article is distributed under the terms of the Creative Commons Attribution License which permits any use, distribution, and reproduction in any medium, provided the original author(s) and source are credited. 